\title{
Non-Apoptotic Role of Apoptotic Caspases in the Drosophila Nervous System
}

\author{
Sarah Colon-Plaza and Tin Tin Su* \\ Department of Molecular, Cellular and Developmental Biology, University of Colorado, Boulder, CO, United States
}

An increasing number of studies demonstrate that cells can activate apoptotic caspases but not die and, instead, display profound changes in cellular structure and function. In this minireview, we will discuss observations in the nervous system of Drosophila melanogaster that illustrate non-apoptotic roles of apoptotic caspases. We will preface these examples with similar observations in other experimental systems and end with a discussion of how apoptotic caspase activity might be constrained to provide non-lethal functions without killing the cell.

Keywords: Drosophila, apoptosis, caspase, central nervous system, peripheral nervous system

\section{INTRODUCTION}

OPEN ACCESS

Edited by:

Bertrand Joseph,

Karolinska Institutet (KI), Sweden

Reviewed by:

Estee Kurant,

University of Haifa, Israel

Luis Alberto Baena-Lopez,

University of Oxford, United Kingdom

*Correspondence:

Tin Tin Su

tin.su@colorado.edu

Specialty section:

This article was submitted to

Signaling,

a section of the journal

Frontiers in Cell and Developmental

Biology

Received: 19 December 2021

Accepted: 14 January 2022

Published: 09 February 2022

Citation:

Colon-Plaza S and Su TT (2022) NonApoptotic Role of Apoptotic Caspases in the Drosophila Nervous System. Front. Cell Dev. Biol. 10:839358. doi: 10.3389/fcell.2022.839358
Caspases are proteases that exist as multi-member families in metazoan numbering, for example, twelve in human and seven in Drosophila melanogaster (Denton et al., 2013; Kesavardhana et al., 2020). Caspase-like proteins have been identified also in fungi and plants (Minina et al., 2017). Some members of the caspase family, for example human caspases 1 and 4 and Drosophila Death related ced-3/Nedd2-like caspase (Dredd), have dedicated roles in non-apoptotic processes such as inflammation and immunity. Other members, for example human caspases 3 and 9 and Drosophila Death regulator Nedd2-like caspase (Dronc) and Death related ICE-like caspase (Drice), are essential for apoptosis and will be referred to as "apoptotic caspases." An increasing body of literature, however, documents non-apoptotic functions of apoptotic caspases, in altering cell identity, sub-cellular remodeling, and production of extra-cellular signals, to name a few. Such apoptotic caspase-driven alterations in cellular structure and function are found in different cell types and across diverse organisms. Here, we will use examples from the Drosophila nervous system to illustrate multiple non-apoptotic roles of apoptotic caspases. We will spring-board off excellent reviews on the subject [for example, (Yamaguchi and Miura, 2015; Melzer and Broemer, 2016; Nakajima and Kuranaga, 2017)], to focus on primary papers published since the publications of these reviews and provide an up-to-date summary.

\section{ACTIVATION OF APOPTOTIC CASPASES}

Caspases are cysteine proteases, that is, they require a cysteine in the active site for activity. Caspases are produced as inactive proenzymes that become activated upon proteolytic cleavage. Activation of apoptotic caspases occurs in a cascade that begins with internal or external death stimuli such as DNA damage. In Drosophila, exposure to ionizing radiation (IR) leads to transcriptional activation of pro-apoptotic proteins Head Involution Defective (Hid) and 


\begin{tabular}{|l|l|l|l|}
\hline $\begin{array}{l}\text { Drosophila } \\
\text { protein }\end{array}$ & $\begin{array}{l}\text { Mammalian } \\
\text { homolog }\end{array}$ & Molecular activity & $\begin{array}{l}\text { Relationship to } \\
\text { apoptosis }\end{array}$ \\
\hline Hid, Rpr & Smac/DIABLO & Protein-binding & Binds and inhibits IAP \\
\hline Dark & Apaf-1 & Protein-binding & $\begin{array}{l}\text { Forms apoptosome to } \\
\text { activate Dronc }\end{array}$ \\
\hline Diap1 & IAP & E3 Ubiquitin ligase, & Inhibits Dronc \\
\hline pronc & Caspase 9 & Protease & $\begin{array}{l}\text { Cleaves and activates } \\
\text { effector caspases }\end{array}$ \\
\hline Drice, Dcp1 & Caspase 3/7 & Protease & $\begin{array}{l}\text { Cleaves substrates to } \\
\text { execute apoptosis }\end{array}$ \\
\hline Numb & Numb & Protein-binding & Binds Dronc \\
\hline Sgg & GSK3 & Kinase & $\begin{array}{l}\text { Target of cleavage by } \\
\text { Drice }\end{array}$ \\
\hline Eiger & TNF & Receptor-binding & $\begin{array}{l}\text { Induced in a Dronc- } \\
\text { dependent manner } \\
\text { after UV damage }\end{array}$ \\
\hline
\end{tabular}

\section{B Hid, Rpr}

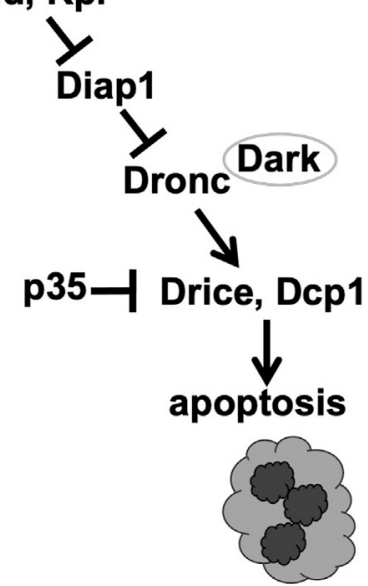

FIGURE 1 | Apoptotic and related proteins discussed in this review. (A) The names of Drosophila and mammalian homologs, along with the known functions. (B) The apoptotic signaling pathway. Only the components discussed in this minireview are shown.

Reaper (Rpr) (Brodsky et al., 2004; Wichmann et al., 2006) (Figure 1). These proteins antagonize Death-associated inhibitor of apoptosis 1 (Diap1) to result in the release and activation by cleavage of Dronc at the apoptosome, a multiprotein structure formed by Death-associated APAF1-related killer (Dark) [reviewed in White et al. (2017)]. Dronc is an apical/initiator caspase that in turn cleaves to activate effector/executioner caspases Drice and Death caspase 1 (Dcp1). Genetic analysis demonstrates that Dronc and Drice are required for DNA damage-induced apoptosis while Dcp1 finetunes this process to accelerate the onset of apoptosis (Florentin and Arama, 2012). Viral caspase inhibitor p35 inhibits effector caspase activity but not initiator caspase activity (Meier et al., 2000; Yoo et al., 2002), and has been used to distinguish the requirements for these two classes of apoptotic caspases.

\section{DROSOPHILA NERVOUS SYSTEMS}

The Central and Peripheral Nervous Systems (CNS and PNS) of a newly hatched Drosophila larva are generated from the embryonic neuroectoderm through sequential cell fate specification events (Singhania and Grueber, 2014; Crews, 2019). Briefly, clusters of cells in the embryonic ectoderm first acquire neuronal competence through the expression of proneural transcription factors. Neurogenic factors such as Notch and Delta then specify a single neuronal precursor cell within each cluster. Neuronal precursor and selector genes then allow neuronal precursors to differentiate into neuronal progenitor cells. Neuronal progenitors are known by different names depending on whether they are in the CNS or PNS and what types of neurons they produce. For example, CNS progenitors are called neuroblasts while PNS progenitors that produce 


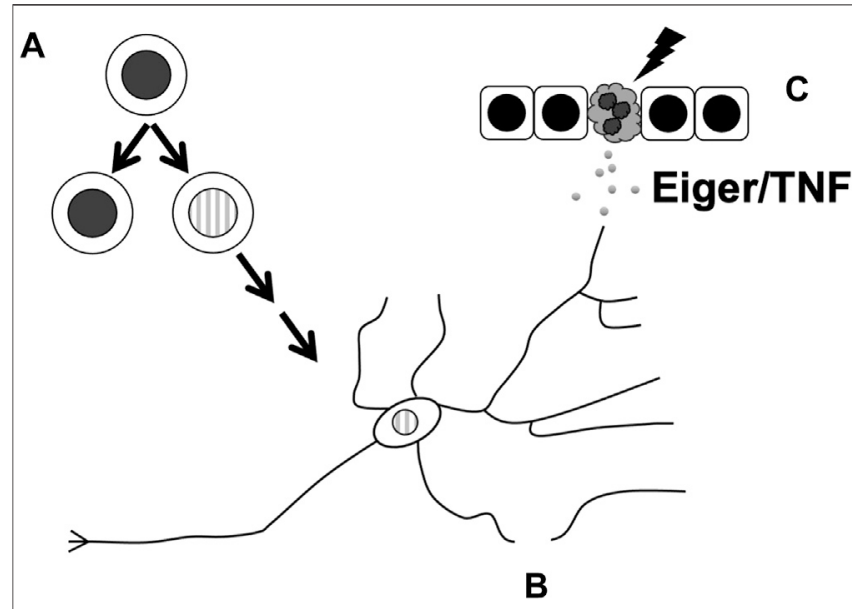

FIGURE 2|Cellular processes in the Drosophila nervous system that are affected by non-apoptotic activities of apoptotic caspases. These include (A) limiting neuronal progenitor cell number by ensuring the fidelity of cell fate specification in the PNS or asymmetric divisions in the CNS, (B) subcellular remodeling during the pruning of dendrites, and (C) facilitating the release of Eiger/TNF from UV-damaged epidermis to result in sensitization of nociceptor neurons.

extrasensory organs are called Sensory Organ Precursors (SOPs). Neuronal progenitor cells undergo stereotypical asymmetric cell divisions to produce two different daughter cells. Ensuring different identities in the cellular progeny requires Numb protein, which is partitioned to just one of the two products of cell division (Rhyu et al., 1994). Numb is a membrane-bound protein with no known biochemical activity that functions through protein-protein interactions. In the progeny of a neuroblast that received Numb, for example, it inhibits Notch signaling to allow differentiation while the other progeny retains the neuroblast identity. Thus, Numb helps limit the number of progenitor cells to prevent hyperproliferation in the CNS. Neuronal cell number is further limited by Programmed Cell Death, an integral part of CNS development that kills by apoptosis approximately one third of CNS cells that are ever born (Crews, 2019). In this context of massive apoptosis, apoptotic caspases provide activities that do not result in cell death as described next.

\section{Changing Cellular Identity}

Metazoan development requires cells to go through several identity changes as they transition from progenitor to a fully differentiated state. Given that the genome remains unchanged, developmental changes in cellular identity must occur through interconnected changes in the transcriptome and in the proteome. As proteases, apoptotic caspases can alter the proteome as seen in numerous examples. Transcription factor Paired Box 7 (Pax7) maintains muscle stem cell identify but its cleavage by apoptotic caspases allows differentiation into myoblasts, a step inhibited by a caspase resistant form of Pax7 (Dick et al., 2015). Caspase 3 null mutant (caspase $3^{-1-}$ ) mice that survive to near-birth stages show reduced skeletal muscle mass. Isolated myoblasts from such mice failed to differentiate into myotubes in culture, indicating a requirement for caspase activity also in a late step in muscle differentiation (Fernando et al., 2002). This requirement may be through Mammalian Sterile Twentylike kinase (MST1) because it includes caspase consensus sites and a shortened MST1 that mimics the cleaved product rescued differentiation in caspase 3 null mutant cells (Fernando et al., 2002; Murray et al., 2008). Additional instances of cellular differentiation, in either normal development or regeneration, show a requirement for apoptotic caspase activity without accompanying evidence for apoptosis [for example, (Fernando et al., 2002; Fernando et al., 2005; Koto et al., 2009),]. Caspase substrates that must be cleaved to allow differentiation include pluripotent factors such as Nanog Homeobox (Nanog) in mouse embryonic stem cells (Fujita et al., 2008) and Abnormal Cell Lineage 29 (Lin-29) during C. elegans larval development (Rougvie and Moss, 2013; Weaver et al., 2014). Likewise, apoptotic caspases contribute to two instances of cellular identity change in the Drosophila nervous system as seen in the following examples.

In the first example, apical caspase Dronc binds Numb to prevent the hyperproliferation of neuroblasts (Ouyang et al., 2011) (Figure 2A). As described above, partitioning of Numb to just one of two cells produced during an asymmetric neuroblast division allows the said recipient to differentiate. Without Numb, both daughter cells retain their progenitor status, leading to ectopic neuroblast formation (ENF) and hyperplasia. A yeast 2-hyrid screen identified Dronc as an interactor of Numb, and Dronc overexpression rescued the ENF phenotype caused by the expression of a dominantnegative numb mutant. This rescue required the presence of wild type Numb, suggesting that the rescue occurs through sequestration of mutant Numb to allow the wildtype Numb to work. The rescue did not require effector caspase activity or the catalytic activity of Dronc, suggesting that Dronc acts in a nonapoptotic manner. Full length Dronc binds Numb as well as cleaved active Dronc, suggesting that any activity provided by Dronc to limit neuroblast number can occur without fear of killing the cell. dronc loss-of-function mutants do not have extra neuroblasts but exacerbate the milder ENF phenotype of weak dominant negative numb mutants. Therefore, Dronc appears to provide an important but redundant function to limit neuroblast number.

Apoptotic caspase activity also limits the number of SOPs, progenitor cells in the PNS (Figure 2A). A subset of SOPs produce external sensory organs called macrochaetes that are readily visible on the back of the adult fly, specifically, in a triangular region called the scutellum. Mutations in dark or dronc as well as inhibition of caspase activity with a dominant-negative Dronc $\left(\right.$ Dronc $^{D N}$ ) or p35 in the scutellum produced extra SOPs and extra macrochaetes. One possible explanation for this phenotype is that caspases normally reduce SOP cell numbers through apoptosis. But this possibility was rendered unlikely by the absence of TUNELpositive cells in the pro-neural clusters that produce the SOPs. Instead, caspases may suppress Wingless (Wg, Drosophila Wnt1) signaling to limit the SOP number; a mutant allele of $w g$ suppressed the extra macrochaetes phenotype that results from 
$\operatorname{Dronc}^{D N}$ while null mutants in Wg inhibitor shaggy (sgg, encoding Drosophila GSK3 kinase) enhanced this phenotype. Sgg contains two caspase $3 / 7$ consensus sites. Expression of Sgg with caspase consensus sites mutated produced extra SOPs and macrochaetes, leading to the model that caspases cleave to activate Sgg, which then opposes Wg to limit the SOP number (Kanuka et al., 2005). As the non-cleavable Sgg produced a phenotype in the presence of endogenous wild type Sgg, the former was proposed to act as a dominant negative against the latter. How might caspase activity finetune SOP number without killing the cells? Additional studies provide evidence that Drosophila I-kappaB kinase (IKK)-like kinase regulates the turnover of Diap1 to temper caspase activity and enables it to provide a developmental role without inducing cell death (Kuranaga et al., 2006; Koto et al., 2009).

While these studies provide compelling evidence for a nonapoptotic role of apoptotic caspases in Drosophila PNS, the data also suggest complexities. For instance, non-cleavable Sgg was less effective than inhibition of Dark or Dronc in producing extra macrochaetes, leading the authors to suggest additional substrates besides Sgg at play (Kanuka et al., 2005). A more recent paper addressed this possibility directly by using CRISPR to replace endogenous Sgg with non-cleavable Sgg (Wang and Baker, 2019). The resulting flies, surprisingly, show normal SOP and macrochaete numbers. These results can be reconciled if noncleavable Sgg acts not by opposing endogenous Sgg but another protein that is the bona fide caspase substrate in controlling SOP number. In other words, while the data support the conclusion that apoptotic caspases play a non-apoptotic role in controlling SOP number, we may not yet know their relevant substrates in this process. The newer study identified an additional upstream regulator of non-apoptotic caspase function in limiting SOP number. Inhibition with mutations or RNAi of expanded (ex), which encodes for a signal transducer in the Hippo (Hpo) pathway, produced extra macrochaetes as did over-expression of transcription factor Yorki (Yki, Drosophila YAP), an inhibitory target of Hpo signaling. The macrochaetes phenotype of ex mutants was rescued by reducing diap1, a known transcriptional target of Yki (Wang and Baker, 2019). These results suggest that $\mathrm{Hpo} / \mathrm{Yki}$ tumor suppressor pathway regulates Diap1 transcriptionally in SOP number determination. This mechanism could operate in parallel with the IKK-mediated mechanism that controls Diap1 protein turnover (Kanuka et al., 2005).

\section{Sub-Cellular Sculpting}

During apoptosis execution, caspase activity helps destroy organelles such as the nucleus and the Golgi (Chiu et al., 2002; Al-Ghorbani et al., 2016). Likewise, caspase activity helps eliminate organelles in some instances of cellular remodeling that accompany differentiation. The removal of the nucleus from the lens cells for light transmission and from red blood cells for efficient oxygen transport requires caspases (Wride et al., 1999; Zermati et al., 2001). In Drosophila, Drice is needed to reduce the cytoplasm and associated organelles during the final step of sperm differentiation (Arama et al., 2003). Inhibiting effector caspase activity with p35 prevented cytoplasm elimination and produced sterile males, illustrating the importance of caspase activity in sperm development. The following examples from two sets of neurons illustrate that subcellular sculpting by apoptotic caspases occurs also in the Drosophila PNS (Figure 2B).

Larval PNS neurons prune their dendrites as the larva metamorphoses into an adult. A genetic screen identified ubcD1, an E2 ubiquitin-conjugating enzyme, as a requirement for pruning in C4da neurons (Kuo et al., 2006). A relevant target of this degradation system appears to be Diap1; a diap1 mutant that was an inefficient substrate for degradation acted as a dominant negative to inhibit pruning. dronc mutants show pruning defects, suggesting that degradation of Diap1 allows Dronc activation and pruning. How is cell death avoided in such a scenario? An antibody against cleaved human caspase 3 shows signal only in dendrites and not in axons and only during the period of dendrite severing. Thus, Diap1 may be degraded locally to produce localized caspase activity that affects localized subcellular remodeling without endangering the cell. Interestingly, expression of $\mathrm{p} 35$, which inhibits effector caspases, did not affect pruning, suggesting that apical caspase activity but not effector caspase activity promotes pruning in C4da neurons. In $\mathrm{ddaC}$ neurons, a genetically encoded caspase activity reporter shows spatially-restricted caspase activation in dendrites undergoing pruning (Williams et al., 2006). Overexpression of Diap1, Dronc ${ }^{\mathrm{DN}}$ or p35 inhibited pruning in ddaC neurons suggesting that both apical and effector caspase activities are needed to prune these neurons. Global caspase activation by overexpression of hid resulted in $\mathrm{ddaC}$ cell death (Williams et al., 2006), providing a contrast to localized caspase activation that allows pruning without cell death.

A more recent study shows that apoptotic caspases play a nonapoptotic role in a phenomenon called neuroprotection that occurs during axonal regeneration (Chen et al., 2016). Briefly, severing of an axon (axotomy) in Drosophila sensory or motor neurons results in neuroprotection (NP) that acts through Jun N-terminal kinase (JNK) and Mitogen-Activated Protein kinase (MAPK). NP can be visualized as preservation of severed dendrites following axotomy and is proposed to buy neurons time to maintain structure and function through the period of axon regeneration. RNAi against dronc or mutations in drice increased the fraction of dendrites protected in ddaE neurons, suggesting that these caspases normally contribute to the removal of severed dendrites, much like in pruning, and must be overcome for NP. The authors then tested the hypothesis that uncontrolled NP might interfere with subsequent axon regeneration. Indeed, neurons with dronc RNAi showed reduced regeneration, leading to the conclusion that Dronc activity promotes axon regeneration by limiting NP.

Neuronal pruning by localized apoptotic caspase activity in Drosophila has parallels in mice where proteasome activity and localized expression of an IAP protein keep the activity of caspases 3 and 9 away from the cell body and restricted to axons to be pruned (Cusack et al., 2013).

\section{Sub-Cellular House-Cleaning}

Fungi and plants encode metacaspases, which are proteases that share similar tertiary structure and an active-site cystine with 
caspases but cleave after Arg or Lys instead of Asp [reviewed in (Minina et al., 2017)]. Budding yeast metacaspase YCA1 is dispensable for cell death (Hauptmann et al., 2006; Kang et al., 2008; Palermo et al., 2013) but ycal mutants show elevated stress response proteins, cytoplasmic protein aggregates and reduced replicative lifespan (Lee et al., 2010; Hill et al., 2014). These data suggest a non-lethal role for a metacaspase in removing harmful protein aggregates from the cytoplasm. Paradoxically, apoptotic caspases may provide an opposite function, to clutter rather than to clean, in Alzheimer's disease $(\mathrm{AD})$ where microtubule-binding protein Tau forms aggregates called neurofibrillary tangles (NFT) (Medeiros et al., 2011). Several lines of data suggest that cleavage of Tau by apoptotic caspases leads to NFT. Tau bears a caspase 3-consensus site and the caspase-cleaved form of Tau as well as cleaved (active) caspase 9 are detected with specific antibodies in neurons from $\mathrm{AD}$ patients but not non-AD controls (Rohn et al., 2002; Gamblin et al., 2003). In mouse disease models, activation of effector caspases precedes NFT formation and caspase-cleaved Tau is sufficient to promote NFT formation in wild type brains (De Calignon et al., 2010). Finally, caspase-cleaved Tau forms fibrils more rapidly than full length Tau in vitro (Gamblin et al., 2003). Collectively, these data lead to the model that apoptosis caspase activity that is not immediately followed by cell death results in Tau NFTs and disease.

A Drosophila model of $\mathrm{AD}$ demonstrates a non-lethal role of apical caspase Dronc in Tau cleavage and enabled an investigation of the observed correlation between circadian dysregulation and susceptibility to neurodegeneration (Means et al., 2015). Disruption of circadian rhythm by RNAi-mediated knock-down of circadian kinase encoded by double time $(d b t)$ or its genetic interactor spaghetti in Drosophila clock neurons led to Dronc activation as detected with an antibody against the cleaved form. Dronc activation in these experiments was observed only during daytime or after light exposure during the night, could spread to nearby non-clock cells, and increased with fly age. Expression of a dominant-negative $d b t$ in the Drosophila eye led to Dronc activation in this tissue, cleavage of co-expressed human Tau in a Droncdependent manner, and neurodegeneration phenotypes. These results in Drosophila parallel the relationship between apoptotic caspase activity, Tau and neurodegenerative disease observed in mammals as described in the preceding paragraph. Furthermore, Drosophila studies add to this picture by identifying Dronc activation as a possible mechanism that links circadian dysregulation with susceptibility to neurodegeneration.

\section{Signaling to Other Cells}

Studies in mice and Drosophila show that dying cells send mitogenic signals that promote tissue homeostasis. In Drosophila larval wing imaginal discs, Dronc promotes Apoptosis-induced Proliferation (AiP) through different mechanisms. First, Dronc acts together with JNK to produce $\mathrm{Wg}$ that acts as a secreted mitogen (Perez-Garijo et al., 2004; Ryoo et al., 2004; Kondo et al., 2006). Second, Dronc activation leads to the elevation of extracellular Reactive Oxygen Species, which recruits macrophages that secrete Eiger/Tumor Necrosis Factor (TNF) to activate JNK and sustain mitogenic signaling (Fogarty et al., 2016; Khan et al., 2017). The role of Dronc in AiP is distinct from its role in activating effector caspases for apoptosis because AiP still occurs when the effector caspases are inhibited. In mice, effector caspases $3 / 7$ cleave and activate Calciumindependent phospholipase A2 to result in the generation and release of prostaglandin $\mathrm{E}$, a known promoter of cell proliferation (Li et al., 2010; Huang et al., 2011). Thus, apoptotic caspase activity produces non-cell autonomous mitogenic signaling in both Drosophila and mammals, albeit through different downstream targets.

A parallel story has emerged recently in Drosophila thermal nociception (sensing painful heat) where Dronc and Eiger function in a non-apoptotic manner to promote sensitization to pain (Jo et al., 2017) (Figure 2C). The exposure of Drosophila larval epidermis to UV results in both apoptosis and allodynia (extreme sensitivity to pain). The latter outcome can be observed as an adverse response to thermal stimuli that would not elicit a response in non-irradiated animals. Dronc is required for both UV-induced apoptosis and thermal allodynia while effector caspases Drice and Dcp1 are required only for apoptosis, suggesting that Dronc plays a non-apoptotic role to induce allodynia. This idea is supported by the finding that doses of UV that are too low to induce apoptosis still induced thermal allodynia in a Droncdependent manner. Dronc, it was found, acts in the epidermis to produce Eiger/TNF that then signals through TNF Receptor (TNFR) on sensory neurons to induce thermal allodynia. Induction of Dronc in the epidermis, even when effector caspases are inhibited to prevent Dronc-induced apoptosis, results in thermal allodynia in an Eigerdependent manner without UV exposure, further lending evidence to a non-apoptotic role for this apoptotic caspase.

\section{REMAINING QUESTIONS}

Given that activation of apoptotic caspases can lead to apoptosis, how do cells restrict this activity for other purposes without being killed? Two mechanisms emerge from the examples described here: keeping caspase activity too low for apoptosis or restricting it spatially. For both mechanisms, regulatory inputs appear to integrate at the level of Diap1, an E3 ubiquitin-ligase that inhibits Dronc via mono- or poly-ubiquitination (Lee et al., 2011; Kamber Kaya et al., 2017). For the first mechanism, IKK-dependent regulation of Diap1 degradation and Hpo/Yki-dependent regulation of Diap1 transcription could keep caspase activity below the threshold for apoptosis yet sufficient to regulate SOP number. For the second mechanism, spatial restriction of Diap1 degradation in C4da neurons may be what allows localized caspase activation and dendrite pruning without killing the cell. Other mechanisms besides regulation of Diap1 likely exist but a comprehensive identification of such mechanisms would require the ability to identify cells that activated apoptotic caspases but did not die. Two recently-described biosensors are helping in this regard. Caspase Tracker and CasExpress rely on 
caspase-mediated recombination events that result in permanent GFP expression, marking cells with past apoptotic caspases activity as well as their clonal descendants (Tang et al., 2015; Ding et al., 2016). These reporters reveal many cell types, including those in the neuronal lineage, that activate apoptotic effector caspases but do not die during Drosophila development. An RNAi screen using a version of the CasExpress reporter identified genes that altered the number of living cells with past caspase activity (Sun et al., 2020). Some of these genes act downstream of or in parallel to caspase activation to decide whether a cell that activated apoptotic caspases will survive or not. One of these genes encodes a homolog of human CDKN1Ainteracting zinc finger protein 1 (CIZ1); how CIZ1 functions to preserve cells that activated caspases remains to be determined. It would be interesting to see if CIZ1 or other regulators of cell survival after caspase activation identified using the CasExpress reporter play a role in the nervous system.

\section{REFERENCES}

Al-Ghorbani, M., Thirusangu, P., Gurupadaswamy, H. D., Girish, V., Shamanth Neralagundi, H. G., Prabhakar, B. T., et al. (2016). Synthesis and Antiproliferative Activity of Benzophenone Tagged Pyridine Analogues towards Activation of Caspase Activated DNase Mediated Nuclear Fragmentation in Dalton's Lymphoma. Bioorg. Chem. 65, 73-81. doi:10. 1016/j.bioorg.2016.02.001

Arama, E., Agapite, J., and Steller, H. (2003). Caspase Activity and a Specific Cytochrome C Are Required for Sperm Differentiation in Drosophila. Dev. Cel 4, 687-697. doi:10.1016/s1534-5807(03)00120-5

Brodsky, M. H., Weinert, B. T., Tsang, G., Rong, Y. S., Mcginnis, N. M., Golic, K. G., et al. (2004). Drosophila melanogaster MNK/Chk2 and P53 Regulate Multiple DNA Repair and Apoptotic Pathways Following DNA Damage. Mol. Cel Biol 24, 1219-1231. doi:10.1128/mcb.24.3.1219-1231.2004

Chen, L., Nye, D. M., Stone, M. C., Weiner, A. T., Gheres, K. W., Xiong, X., et al. (2016). Mitochondria and Caspases Tune Nmnat-Mediated Stabilization to Promote Axon Regeneration. Plos Genet. 12, e1006503. doi:10.1371/journal. pgen.1006503

Chiu, R., Novikov, L., Mukherjee, S., and Shields, D. (2002). A Caspase Cleavage Fragment of P115 Induces Fragmentation of the Golgi Apparatus and Apoptosis. J. Cel Biol 159, 637-648. doi:10.1083/jcb.200208013

Crews, S. T. (2019). Drosophila Embryonic CNS Development: Neurogenesis, Gliogenesis, Cell Fate, and Differentiation. Genetics 213, 1111-1144. doi:10. 1534/genetics.119.300974

Cusack, C. L., Swahari, V., Hampton Henley, W., Michael Ramsey, J., and Deshmukh, M. (2013). Distinct Pathways Mediate Axon Degeneration during Apoptosis and Axon-specific Pruning. Nat. Commun. 4, 1876. doi:10.1038/ncomms2910

De Calignon, A., Fox, L. M., Pitstick, R., Carlson, G. A., Bacskai, B. J., Spires-Jones, T. L., et al. (2010). Caspase Activation Precedes and Leads to Tangles. Nature 464, 1201-1204. doi:10.1038/nature08890

Denton, D., Aung-Htut, M. T., and Kumar, S. (2013). Developmentally Programmed Cell Death in Drosophila. Biochim. Biophys. Acta (Bba) - Mol. Cel Res. 1833, 3499-3506. doi:10.1016/j.bbamcr.2013.06.014

Dick, S. A., Chang, N. C., Dumont, N. A., Bell, R. A. V., Putinski, C., Kawabe, Y., et al. (2015). Caspase 3 Cleavage of Pax7 Inhibits Self-Renewal of Satellite Cells. Proc. Natl. Acad. Sci. USA 112, E5246-E5252. doi:10.1073/pnas.1512869112

Ding, A. X., Sun, G., Argaw, Y. G., Wong, J. O., Easwaran, S., and Montell, D. J. (2016). CasExpress Reveals Widespread and Diverse Patterns of Cell Survival of Caspase-3 Activation during Development In Vivo. Elife 5. doi:10.7554/eLife.10936

Fernando, P., Brunette, S., and Megeney, L. A. (2005). Neural Stem Cell Differentiation Is Dependent upon Endogenous Caspase-3 Activity. FASEB j. 19, 1671-1673. doi:10.1096/fj.04-2981fje

\section{AUTHOR CONTRIBUTIONS}

SC-P and TS performed literature search and co-drafted the manuscript. TS edited and revised the manuscript.

\section{FUNDING}

TS is supported by a grant from the National Institutes of Health (R35 GM130374). SC-P is supported by an administrative supplement to the same grant (R35 GM130374-S1).

\section{ACKNOWLEDGMENTS}

The authors thank the members of the Su Lab for critical reading of the manuscript.

Fernando, P., Kelly, J. F., Balazsi, K., Slack, R. S., and Megeney, L. A. (2002). Caspase 3 Activity Is Required for Skeletal Muscle Differentiation. Proc. Natl. Acad. Sci. 99, 11025-11030. doi:10.1073/pnas.162172899

Florentin, A., and Arama, E. (2012). Caspase Levels and Execution Efficiencies Determine the Apoptotic Potential of the Cell. J. Cel Biol 196, 513-527. doi:10. 1083/jcb.201107133

Fogarty, C. E., Diwanji, N., Lindblad, J. L., Tare, M., Amcheslavsky, A., Makhijani, K., et al. (2016). Extracellular Reactive Oxygen Species Drive ApoptosisInduced Proliferation via Drosophila Macrophages. Curr. Biol. 26, 575-584. doi:10.1016/j.cub.2015.12.064

Fujita, J., Crane, A. M., Souza, M. K., Dejosez, M., Kyba, M., Flavell, R. A., et al. (2008). Caspase Activity Mediates the Differentiation of Embryonic Stem Cells. Cell Stem Cell 2, 595-601. doi:10.1016/j.stem.2008.04.001

Gamblin, T. C., Chen, F., Zambrano, A., Abraha, A., Lagalwar, S., Guillozet, A. L., et al. (2003). Caspase Cleavage of Tau: Linking Amyloid and Neurofibrillary Tangles in Alzheimer's Disease. Proc. Natl. Acad. Sci. 100, 10032-10037. doi:10. 1073/pnas.1630428100

Hauptmann, P., Riel, C., Kunz-Schughart, L. A., Frohlich, K.-U., Madeo, F., and Lehle, L. (2006). Defects in N-Glycosylation Induce Apoptosis in Yeast. Mol. Microbiol. 59, 765-778. doi:10.1111/j.1365-2958.2005.04981.x

Hill, S. M., Hao, X., Liu, B., and Nyström, T. (2014). Life-span Extension by a Metacaspase in the Yeast Saccharomyces cerevisiae. Science 344, 1389-1392. doi:10.1126/science.1252634

Huang, Q., Li, F., Liu, X., Li, W., Shi, W., Liu, F.-F., et al. (2011). Caspase 3mediated Stimulation of Tumor Cell Repopulation during Cancer Radiotherapy. Nat. Med. 17, 860-866. doi:10.1038/nm.2385

Jo, J., Im, S. H., Babcock, D. T., Iyer, S. C., Gunawan, F., Cox, D. N., et al. (2017). Drosophila Caspase Activity Is Required Independently of Apoptosis to Produce Active TNF/Eiger during Nociceptive Sensitization. Cell Death Dis 8, e2786. doi:10.1038/cddis.2016.474

Kamber Kaya, H. E., Ditzel, M., Meier, P., and Bergmann, A. (2017). An Inhibitory Mono-Ubiquitylation of the Drosophila Initiator Caspase Dronc Functions in Both Apoptotic and Non-apoptotic Pathways. Plos Genet. 13, e1006438. doi:10. 1371/journal.pgen.1006438

Kang, M.-S., Lee, S.-K., Park, C.-S., Kang, J.-H., Bae, S.-H., and Yu, S.-L. (2008). Expression of Death Receptor 4 Induces Caspase-independent Cell Death in MMS-Treated Yeast. Biochem. Biophysical Res. Commun. 376, 305-309. doi:10. 1016/j.bbrc.2008.08.159

Kanuka, H., Kuranaga, E., Takemoto, K., Hiratou, T., Okano, H., and Miura, M. (2005). Drosophila Caspase Transduces Shaggy/GSK-3 $\beta$ Kinase Activity in Neural Precursor Development. EMBO J. 24, 3793-3806. doi:10.1038/sj.emboj. 7600822

Kesavardhana, S., Malireddi, R. K. S., and Kanneganti, T.-D. (2020). Caspases in Cell Death, Inflammation, and Pyroptosis. Annu. Rev. Immunol. 38, 567-595. doi:10.1146/annurev-immunol-073119-095439 
Khan, S. J., Abidi, S. N. F., Skinner, A., Tian, Y., and Smith-Bolton, R. K. (2017). The Drosophila Duox Maturation Factor Is a Key Component of a Positive Feedback Loop that Sustains Regeneration Signaling. Plos Genet. 13, e1006937. doi:10.1371/journal.pgen.1006937

Kondo, S., Senoo-Matsuda, N., Hiromi, Y., and Miura, M. (2006). DRONC Coordinates Cell Death and Compensatory Proliferation. Mol. Cel Biol 26, 7258-7268. doi:10.1128/mcb.00183-06

Koto, A., Kuranaga, E., and Miura, M. (2009). Temporal Regulation of Drosophila IAP1 Determines Caspase Functions in Sensory Organ Development. J. Cel Biol 187, 219-231. doi:10.1083/jcb.200905110

Kuo, C. T., Zhu, S., Younger, S., Jan, L. Y., and Jan, Y. N. (2006). Identification of E2/E3 Ubiquitinating Enzymes and Caspase Activity Regulating Drosophila Sensory Neuron Dendrite Pruning. Neuron 51, 283-290. doi:10.1016/j.neuron.2006.07.014

Kuranaga, E., Kanuka, H., Tonoki, A., Takemoto, K., Tomioka, T., Kobayashi, M., et al. (2006). Drosophila IKK-Related Kinase Regulates Nonapoptotic Function of Caspases via Degradation of IAPs. Cell 126, 583-596. doi:10.1016/j.cell.2006.05.048

Lee, R. E. C., Brunette, S., Puente, L. G., and Megeney, L. A. (2010). Metacaspase Ycal Is Required for Clearance of Insoluble Protein Aggregates. Proc. Natl. Acad. Sci. 107, 13348-13353. doi:10.1073/pnas.1006610107

Lee, T. V., Fan, Y., Wang, S., Srivastava, M., Broemer, M., Meier, P., et al. (2011). Drosophila IAP1-Mediated Ubiquitylation Controls Activation of the Initiator Caspase DRONC Independent of Protein Degradation. Plos Genet. 7, e1002261. doi:10.1371/journal.pgen.1002261

Li, F., Huang, Q., Chen, J., Peng, Y., Roop, D. R., Bedford, J. S., et al. (2010). Apoptotic Cells Activate the "phoenix Rising" Pathway to Promote Wound Healing and Tissue Regeneration. Sci. Signal. 3, ra13. doi:10.1126/scisignal. 2000634

Means, J. C., Venkatesan, A., Gerdes, B., Fan, J.-Y., Bjes, E. S., and Price, J. L. (2015). Drosophila Spaghetti and Doubletime Link the Circadian Clock and Light to Caspases, Apoptosis and Tauopathy. Plos Genet. 11, e1005171. doi:10.1371/ journal.pgen.1005171

Medeiros, R., Baglietto-Vargas, D., and Laferla, F. M. (2011). The Role of Tau in Alzheimer's Disease and Related Disorders. CNS Neurosci. Ther. 17, 514-524. doi:10.1111/j.1755-5949.2010.00177.x

Meier, P., Silke, J., Leevers, S. J., and Evan, G. I. (2000). The Drosophila Caspase DRONC Is Regulated by DIAP1. EMBO J. 19, 598-611. doi:10.1093/emboj/19. 4.598

Melzer, J., and Broemer, M. (2016). Nerve-racking - Apoptotic and Non-apoptotic Roles of Caspases in the Nervous System ofDrosophila. Eur. J. Neurosci. 44, 1683-1690. doi:10.1111/ejn.13213

Minina, E. A., Coll, N. S., Tuominen, H., and Bozhkov, P. V. (2017). Metacaspases versus Caspases in Development and Cell Fate Regulation. Cell Death Differ 24, 1314-1325. doi:10.1038/cdd.2017.18

Murray, T. V. A., Mcmahon, J. M., Howley, B. A., Stanley, A., Ritter, T., Mohr, A., et al. (2008). A Non-apoptotic Role for Caspase-9 in Muscle Differentiation. J. Cel Sci 121, 3786-3793. doi:10.1242/jcs.024547

Nakajima, Y.-i., and Kuranaga, E. (2017). Caspase-dependent Non-apoptotic Processes in Development. Cel Death Differ 24, 1422-1430. doi:10.1038/cdd. 2017.36

Ouyang, Y., Petritsch, C., Wen, H., Jan, L., Jan, Y. N., and Lu, B. (2011). Dronc Caspase Exerts a Non-apoptotic Function to Restrain Phospho-Numb-Induced Ectopic Neuroblast Formation in Drosophila. Development 138, 2185-2196. doi:10.1242/dev.058347

Palermo, V., Mangiapelo, E., Piloto, C., Pieri, L., Muscolini, M., Tuosto, L., et al. (2013). p53 Death Signal Is Mainly Mediated by Nucl(EndoG) in the yeastSaccharomyces Cerevisiae. FEMS Yeast Res. 13, 682-688. doi:10.1111/ 1567-1364.12067

Pérez-Garijo, A., Martín, F. A., and Morata, G. (2004). Caspase Inhibition during Apoptosis Causes Abnormal Signalling and Developmental Aberrations in Drosophila. Development 131, 5591-5598. doi:10.1242/dev.01432

Rhyu, M. S., Jan, L. Y., and Jan, Y. N. (1994). Asymmetric Distribution of Numb Protein during Division of the Sensory Organ Precursor Cell Confers Distinct Fates to Daughter Cells. Cell 76, 477-491. doi:10.1016/0092-8674(94)90112-0
Rohn, T. T., Rissman, R. A., Davis, M. C., Kim, Y. E., Cotman, C. W., and Head, E. (2002). Caspase-9 Activation and Caspase Cleavage of Tau in the Alzheimer's Disease Brain. Neurobiol. Dis. 11, 341-354. doi:10.1006/nbdi.2002.0549

Rougvie, A. E., and Moss, E. G. (2013). Developmental Transitions in C. elegans Larval Stages. Curr. Top. Dev. Biol. 105, 153-180. doi:10.1016/b978-0-12396968-2.00006-3

Ryoo, H. D., Gorenc, T., and Steller, H. (2004). Apoptotic Cells Can Induce Compensatory Cell Proliferation through the JNK and the Wingless Signaling Pathways. Dev. Cel 7, 491-501. doi:10.1016/j.devcel.2004.08.019

Singhania, A., and Grueber, W. B. (2014). Development of the Embryonic and Larval Peripheral Nervous System ofDrosophila. Wires Dev. Biol. 3, 193-210. doi:10.1002/wdev.135

Sun, G., Ding, X. A., Argaw, Y., Guo, X., and Montell, D. J. (2020). Akt1 and dCIZ1 Promote Cell Survival from Apoptotic Caspase Activation during Regeneration and Oncogenic Overgrowth. Nat. Commun. 11, 5726. doi:10.1038/s41467-02019068-2

Tang, H. L., Tang, H. M., Fung, M. C., and Hardwick, J. M. (2015). In Vivo CaspaseTracker Biosensor System for Detecting Anastasis and Non-apoptotic Caspase Activity. Sci. Rep. 5, 9015. doi:10.1038/srep09015

Wang, L.-H., and Baker, N. E. (2019). Salvador-Warts-Hippo Pathway Regulates Sensory Organ Development via Caspase-dependent Nonapoptotic Signaling. Cel Death Dis 10, 669. doi:10.1038/s41419-019-1924-3

Weaver, B. P., Zabinsky, R., Weaver, Y. M., Lee, E. S., Xue, D., and Han, M. (2014). CED-3 Caspase Acts with miRNAs to Regulate Non-apoptotic Gene Expression Dynamics for Robust Development in C. elegans. Elife 3, e04265. doi:10.7554/eLife.04265

White, K., Arama, E., and Hardwick, J. M. (2017). Controlling Caspase Activity in Life and Death. Plos Genet. 13, e1006545. doi:10.1371/journal.pgen.1006545

Wichmann, A., Jaklevic, B., and Su, T. T. (2006). Ionizing Radiation Induces Caspase-dependent but Chk2- and P53-independent Cell Death in Drosophila melanogaster. Proc. Natl. Acad. Sci. 103, 9952-9957. doi:10.1073/pnas. 0510528103

Williams, D. W., Kondo, S., Krzyzanowska, A., Hiromi, Y., and Truman, J. W. (2006). Local Caspase Activity Directs Engulfment of Dendrites during Pruning. Nat. Neurosci. 9, 1234-1236. doi:10.1038/nn1774

Wride, M. A., Parker, E., and Sanders, E. J. (1999). Members of the Bcl-2 and Caspase Families Regulate Nuclear Degeneration during Chick Lens Fibre Differentiation. Dev. Biol. 213, 142-156. doi:10.1006/dbio.1999.9375

Yamaguchi, Y., and Miura, M. (2015). Programmed Cell Death and Caspase Functions during Neural Development. Curr. Top. Dev. Biol. 114, 159-184. doi:10.1016/bs.ctdb.2015.07.016

Yoo, S. J., Huh, J. R., Muro, I., Yu, H., Wang, L., Wang, S. L., et al. (2002). Hid, Rpr and Grim Negatively Regulate DIAP1 Levels through Distinct Mechanisms. Nat. Cel Biol 4, 416-424. doi:10.1038/ncb793

Zermati, Y., Garrido, C., Amsellem, S., Fishelson, S., Bouscary, D., Valensi, F., et al. (2001). Caspase Activation Is Required for Terminal Erythroid Differentiation. J. Exp. Med. 193, 247-254. doi:10.1084/jem.193.2.247

Conflict of Interest: The authors declare that the research was conducted in the absence of any commercial or financial relationships that could be construed as a potential conflict of interest.

Publisher's Note: All claims expressed in this article are solely those of the authors and do not necessarily represent those of their affiliated organizations, or those of the publisher, the editors and the reviewers. Any product that may be evaluated in this article, or claim that may be made by its manufacturer, is not guaranteed or endorsed by the publisher.

Copyright (c) 2022 Colon-Plaza and Su. This is an open-access article distributed under the terms of the Creative Commons Attribution License (CC BY). The use, distribution or reproduction in other forums is permitted, provided the original author(s) and the copyright owner(s) are credited and that the original publication in this journal is cited, in accordance with accepted academic practice. No use, distribution or reproduction is permitted which does not comply with these terms. 